\title{
Calidad docente en el ámbito universitario: Un estudio comparativo de las universidades andaluzas
}

\author{
Pursuing quality in higher education: A comparative analysis of \\ actions taken in andalusian universities
}

\author{
Francisca Castilla Polo (fpolo@ujaen.es) \\ Universidad de Jaén (España) \\ http://dx.doi.org/10.12795/EDUCADE.2011.i02.09
}

\begin{abstract}
RESUMEN: La calidad docente dentro del ámbito universitario es un tema de gran interés en un momento de adaptación al Espacio Europeo de Educación Superior (EEES) como el actual. Por este motivo, nos proponemos ofrecer una visión de conjunto sobre las diferentes actuaciones que las universidades andaluzas están llevando a cabo para reforzar y/o incrementar la calidad de su enseñanza, así como analizar los principios sobre los que se basan tales iniciativas. Mediante la realización de un cuestionario postal, se ha constatado la existencia de ciertas actuaciones que se repiten dentro del ámbito universitario: los cursos de formación del profesorado, los proyectos de innovación docente y las ayudas para la movilidad. Sin embargo, aunque se está apostando por ellas de forma generalizada, hemos observado que no comparten un fondo común basado en principios de calidad como cabría esperar.
\end{abstract}

PALABRAS CLAVE: Calidad de la enseñanza, Profesor universitario, Innovación docente, Espacio Europeo de Educación Superior.

\begin{abstract}
The importance of the education quality in the Higher Education field is a topic object of priority attention even more in an adaptation moment to the European Higher Education Area (EHEA) as is the one which we find. For this reason, set out to offer a set vision on different actions that Andalusian Universities are carrying out to reinforce and/or to increase his education quality as well as analyzing the principles on which they are based. The methodology employed has consisted in a postal questionnaire about these topics. As main contribution of our research we can argue the existence of some repeated actions within the university field for the improvement of the education quality, adverted to training, teaching innovation projects and aids for the mobility. Nonetheless, observe that although is being bet for these initiatives do not share a mutual fund based on quality principles as it fitted to expect.
\end{abstract}

KEYWORDS: Teaching quality, Higher education teachers, Educational innovation, European Higher Education Area.

\section{INTRODUCCIÓN}

La calidad docente en la educación universitaria es un tema de gran importancia en un momento de adaptación al Espacio Europeo de Educación Superior (EEES) como el actual, donde se está implementando una importante reforma universitaria a todos los niveles. No se cuestionan los beneficios que se asocian a una enseñanza de calidad, siendo una exigencia imprescindible para avalar el adecuado funcionamiento de la educación universitaria. Sin embargo, estimamos necesario profundizar en los medios con los que desde el ámbito universitario se está abordando el proceso de su mejora.

Artículo. Recibido: 16-12-09 - Versiones revisadas: 09-04; 10-05-11, Aceptado: 07-06-11

Licencia Creative Commons (CC) BY-NC-ND · Asociación Española de Contabilidad y Administración de Empresas - AECA 
La sociedad en su conjunto reclama que el funcionamiento de las universidades esté vinculado a criterios de eficacia, eficiencia y excelencia cada vez mayores (Arranz, 2007: 34), hecho que implica que la calidad ocupe un eje central en el diseño de la política universitaria. Por este motivo, nos proponemos ofrecer una visión de conjunto sobre las diferentes actuaciones que se están llevando a cabo en el ámbito universitario para reforzar y/o incrementar su calidad docente, así como analizar los principios sobre los que tales iniciativas se basan. Con el término calidad docente queremos hacer referencia a todas aquellas actuaciones destinadas a mejorar los conocimientos, competencias y habilidades del profesor universitario, todas ellas con repercusión inmediata en el aula y donde el papel de la innovación educativa ocupa un lugar imprescindible. Siguiendo a Marqués (2008), podemos incluso afirmar que el EEES ha favorecido el interés hacia métodos pedagógicos innovadores.

Específicamente, nos centraremos en el ámbito de las universidades públicas andaluzas, las cuales disponen de una guía específica de principios y actuaciones recogidas en el "Informe de la Comisión para la Innovación de la Docencia en las Universidades Andaluzas" (CIDUA) (Junta de Andalucía, 2005). Esto nos lleva a establecer como primer objetivo analizar si las diferentes iniciativas llevadas a cabo en materia de calidad docente se basan en este Informe o en otros principios de calidad diferentes.

Nuestro segundo objetivo consiste en revisar la calidad docente desde un punto de vista aplicado. Para ello, consideramos oportuno conocer el órgano de gobierno encargado de esta actividad dentro de la estructura universitaria y concretar las funciones que tiene atribuidas para poder situar la calidad docente en términos organizativos. Con esta información, y el estudio de las actuaciones realizadas para la mejora de la calidad docente dentro de las universidades andaluzas, podremos comparar y contrastar los resultados obtenidos en relación a este tema.

El tercer objetivo de este trabajo analiza si es posible determinar unas actuaciones comunes que conformarían un Modelo Andaluz para la Mejora de la Calidad Docente. Serán fundamentales los resultados obtenidos en este primer acercamiento al caso andaluz para, en una segunda fase, ampliar la investigación iniciada con el análisis del ámbito geográfico nacional.

El método de trabajo incluye tres pasos fundamentales. En primer lugar, efectuaremos una revisión teórica de los estudios, informes y proyectos existentes sobre calidad docente e innovación de la docencia universitaria. A continuación, analizaremos el fondo y la forma en que las universidades andaluzas están abordando la mejora de su calidad docente. Por último, expondremos las conclusiones generales de nuestro estudio y sus limitaciones, así como también plantearemos futuras líneas de investigación.

\section{CALIDAD E INNOVACIÓN DOCENTE EN EL ÁMBITO UNIVERSITARIO}

En este apartado situaremos la importancia de la calidad docente dentro del contexto universitario. De forma más específica, nos centraremos en justificar la necesidad de mejorar la calidad del docente universitario como agente fundamental del proceso de enseñanza-aprendizaje dentro del EESS. Del mismo modo, analizaremos la relación que existe entre calidad docente e innovación, por ser éste un elemento fundamental para entender las iniciativas empleadas por las Universidades para la mejora de su calidad docente. 


\subsection{La Calidad en el Contexto Universitario}

Para Galán (2009:19) "la gestión de la calidad es un requisito que se da por supuesto que realizan las organizaciones y entre ellas las Universidades". Para Villa (2008:180), resulta evidente que la calidad "ha entrado en el lenguaje, en los planes estratégicos universitarios, y en lo que aún es mejor, en la mentalidad de la gran parte del profesorado universitario". "La mejora de calidad refuerza el atractivo de las Universidades Europeas dentro del EEES" (Harvey y Stensaker, 2008:433). No obstante, los pronunciamientos anteriores indican que la cultura de la calidad debería ser un concepto para identificar cambios y no para adaptarse a ellos, cuestión que parece ser la realidad encontrada en el ámbito universitario europeo a la hora de su incorporación al Proceso de Bolonia.

La calidad ocupa un papel estratégico dentro de las diferentes normativas que regulan la actividad de las Universidades Españolas. La Ley Orgánica de Universidades (LOU) modificada en 2007, se refiere entre otras cuestiones en su artículo 31 a que "la promoción y la garantía de la calidad de las Universidades Españolas, en el ámbito nacional e internacional, es un fin esencial de la política universitaria", teniendo lo anterior objetivos como el mencionado en el apdo. c "la mejora de la actividad docente e investigadora y de la gestión de las Universidades". También la Ley Andaluza de Universidades (LAU), de 2003 en su artículo 57 indica que "las universidades andaluzas potenciarán la calidad de la docencia y la investigación como expresión de la actividad universitaria, y concretamente las Universidades otorgarán atención prioritaria a la calidad de la docencia...". De lo anterior se deduce la importancia de que las Universidades apuesten por la mejora de su calidad en términos generales y específicos para su labor docente, aspecto al que nos dedicamos en este trabajo.

Aunque el concepto calidad es difícil de concretar cuando se aplica a la educación superior, según Gvaramadze (2008) se pueden identificar dos vertientes del mismo: la calidad como proceso de mejora y la calidad como transformación individual. En el primer caso, la calidad se asocia a una institución y generalmente se suele basar en su autonomía, transparencia y efectividad, mientras que cuando hablamos de casos individuales, la calidad se asocia a los estudiantes, académicos y plantilla de cada una de esas instituciones, ya sean Universidades o Programas Académicos. Nos centraremos en este trabajo en la calidad académica o calidad docente.

\subsection{La Calidad Docente: Una exigencia del EeES}

El concepto "desarrollo profesional del docente universitario" definido por el Ministerio de Educación y Ciencia en el informe "La formación del profesor universitario" (1992: 35) como "cualquier intento sistemático de cambiar la práctica, creencias y conocimientos profesionales del docente universitario, hacia un propósito de mejora de la calidad docente, investigadora y de gestión", deja sentado el interés hacia la calidad docente y su mejora. No obstante, debemos indicar que mencionado desarrollo profesional hace referencia a un término más amplio y enfocado a la vertiente estratégica universitaria que aquellos tradicionalmente empleados en este ámbito de la carrera profesional, tal y como indica Sánchez (2001:1) "perfeccionamiento del profesorado, formación continua, formación permanente del profesorado, entrenamiento, perfeccionamiento o formación en servicio".

La actividad docente, como función esencial y no única de la actividad universitaria, exige ser realizada con unos mínimos requisitos que garanticen su calidad y más en una etapa de cambios como la actual. "Trabajar en el nuevo modelo universitario exige una mayor implicación del profesorado en la docencia" (Pallisera et alt., 
2010:11). En el otro extremo, también la Universidad debe asumir las directrices indicadas por la Declaración de Bolonia y eso implica la puesta en marcha de un conjunto importante de actuaciones encaminadas hacia la configuración de una nueva relación entre profesores y alumnos basada en el eje del aprendizaje, al igual que la puesta a disposición de medios materiales necesarios para llevar a cabo tal reforma con garantías (Pallisera et alt., 2010:12).

Para la European Association for Quality Assurance in Higher Education (ENQA) en su informe "Standards and Guidelines for Quality Assurance in the European Higher Education Area"(2005), el papel de los docentes vuelve a resultar fundamental en el logro de un EEES de calidad. Concretamente, se refiere a la necesidad de que el profesorado esté satisfecho e implicado en el aprendizaje de sus alumnos debiendo quedar sujeto a evaluaciones externas que acrediten tales competencias. Resultan consideraciones a tener en cuenta en relación con la calidad docente según la ENQA, entre otras, las siguientes: deben darse oportunidades para que desarrollen y amplíen su capacidad de enseñanza y estimularles para que saquen partido a sus habilidades.

Siguiendo a Vizcarro (2003:13), existen una serie de características que identifican a la docencia de calidad: contenidos del curso, características de las presentaciones en clase, gestión del curso, enseñanza fuera del aula, calidad de los aprendizajes, actitud profesional y crítica del docente -Tabla 1-. Entre las anteriores también se incluye la apuesta por la innovación en la docencia como un indicador de calidad necesario.

También a nivel andaluz, el Informe de la CIDUA (Junta de Andalucía, 2005: 43 y ss.) se refiere a un conjunto de principios tendentes a garantizar una docencia universitaria de calidad. Indica explícitamente la necesidad de que ésta sea:

a) Activa, encaminada a resolver los problemas derivados de la práctica docente mediante un proceso de reflexión.

b) Enfocada de manera holística en el desarrollo de competencias profesionales específicas a cada titulación o ámbito profesional.

c) Estimuladora sobre la experiencia, destinada a compartir la práctica docente mediante el proceso de comunicación entre profesores y alumnos.

d) Que tome en consideración las especialidades académicas del alumnado.

e) Que se asocie con la calidad de la institución pues ambos componentes del sistema universitario se complementan.

f) Que permita la conexión entre docencia e investigación dentro del currículum del profesor.

\subsection{La InNOVACIÓn Como Estrategia de CALIdad}

Desde hace algún tiempo Seymour (1988) advertía sobre la importancia de la innovación educativa y su consideración como objetivo prioritario para la educación superior. A nivel andaluz, el Informe CIDUA demanda para abordar la convergencia con Europa "un esfuerzo por mejorar la calidad del servicio público que la Universidad ofrece a través de la innovación de los modos habituales de enseñar y aprender en la Universidad" (Junta de Andalucía, 2005:61). Igualmente, para Mauri et alt. (2004:2) el EEES "ha promovido el desarrollo de cambios educativos relevantes que han supuesto la puesta en marcha de numerosas iniciativas en el ámbito de la innovación de la docencia universitaria". En opinión de estos autores existe una relación directa entre la innovación docente y los principios que deben guiar la docencia de calidad. También mantiene esta posición Galán (2009) al afirmar que cuando una Universidad se instala 
en la cultura de mejora continua de su calidad entonces pasa a ser una organización innovadora.

TABLA 1. Características de UNA doCENCIA DE CALIDAD

\begin{tabular}{|c|c|}
\hline Elemento & Descripción/detalle \\
\hline Contenidos del curso & $\begin{array}{l}\text { Selección y adecuación de contenidos y objetivos } \\
\text { correctamente formulados, organizados y realistas para el tiempo } \\
\text { disponible, precisando los métodos docentes que permitirán } \\
\text { alcanzarlos así como los métodos de evaluación ajustados a los } \\
\text { objetivos. }\end{array}$ \\
\hline $\begin{array}{l}\text { Características de las } \\
\text { presentaciones en clase }\end{array}$ & $\begin{array}{l}\text { Claridad de las explicaciones, capacidad de comunicación, } \\
\text { entusiasmo, habilidad para plantear y responder preguntas, etc. }\end{array}$ \\
\hline Gestión del curso & $\begin{array}{l}\text { Organización docente, métodos de docencia y evaluación, } \\
\text { accesibilidad para los estudiantes, calidad de la } \\
\text { retroalimentación, coordinación con ayudantes u otros profesores } \\
\text { en su caso, organización de actividades para los estudiantes tales } \\
\text { como proyectos o problemas prácticos, visitas de campo, } \\
\text { elaboración de materiales docentes, etc. }\end{array}$ \\
\hline $\begin{array}{l}\text { Enseñanzas fuera de } \\
\text { clase }\end{array}$ & $\begin{array}{l}\text { Tutorización de estudiantes, planificación y coordinación de } \\
\text { asignaturas y programas de estudio, escribir libros de textos y otros } \\
\text { materiales didácticos, etc. }\end{array}$ \\
\hline $\begin{array}{l}\text { Calidad de los } \\
\text { aprendizajes }\end{array}$ & $\begin{array}{l}\text { Como producto final de una docencia de calidad, incluyendo } \\
\text { adquisición de competencias generales y específicas, motivación } \\
\text { de los estudiantes para seguir estudiando la materia u otras } \\
\text { afines, ajuste de los estudiantes egresados a perfiles científicos o } \\
\text { profesionales actualizados, etc. }\end{array}$ \\
\hline $\begin{array}{l}\text { Actitud profesional y } \\
\text { crítica }\end{array}$ & $\begin{array}{l}\text { Ante la docencia, reflexión y planteamiento explícito y continuo } \\
\text { de la docencia, participación en equipos de trabajo de finalidad } \\
\text { docente, autoevaluación, participación en proyectos de } \\
\text { innovación. }\end{array}$ \\
\hline
\end{tabular}

Fuente: Elaboración a partir de Vizcarro (2003:13)

Más detallada es la visión que ofrece Pulido (2007:17) sobre el concepto innovación. En su opinión, la Universidad debe manejar una triple visión de innovación para desarrollar su función: institucional, productiva y espacial. Las dos primeras englobarían aquellas actividades de innovación en áreas transversales como la gobernanza y la financiación, así como también los procesos relacionados con las tres funciones que este autor asocia a la Universidad: la educación, la investigación y la conexión con la sociedad. Con relación a este último ámbito, encontraríamos la innovación para el aprendizaje de la educación superior, para la generación y transferencia de conocimiento científico-tecnológico y para mejorar y/o reforzar las relaciones Universidad-Sociedad. También resulta necesario según este autor incidir en una visión espacial de la innovación que contemple la globalización y la internacionalización universitaria. Véase la llustración $n^{\circ} 1$.

Nos preocupa especialmente en este trabajo la innovación productiva que delimitamos al papel de la innovación docente, entendida al igual que lo hacen Bueno y Escudero (2005:595) como "un proceso de mejora que el profesorado afronta de forma práctica y concreta y con una repercusión inmediata en el aula, un proceso 
de mejora que supone un cambio novedoso de los planteamientos y métodos de su docencia". El fomento de la innovación docente es una de las principales vías, no la única, con las que las Universidades están fomentando la mejora de su calidad docente y a la vez están respondiendo a los nuevos cambios del contexto universitario.

\section{ILUSTRACIÓN 1. LA TRIPLE VISIÓN DE LA INNOVACIÓN UNIVERSITARIA}

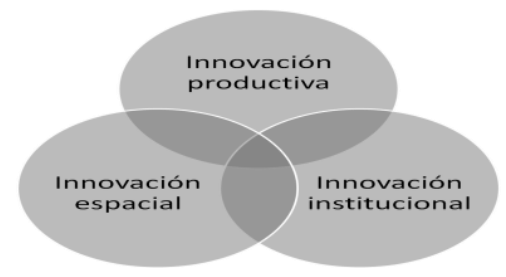

Fuente: Pulido (2007:18)

No consideramos que resulte la única vía porque consideramos que la calidad docente se puede mejorar también con la formación y con la movilidad, aspectos a los que nos referiremos a continuación.

\subsection{Iniciativas para la Mejora de la Calidad Docente}

Una vez que han sido presentados los conceptos fundamentales de nuestro trabajo, el contexto del EEES y el papel de la innovación, dedicamos este último apartado a revisar desde un punto de vista teórico las iniciativas más utilizadas para mejorar la calidad docente en el ámbito universitario.

Generalmente siempre se ha planteado una relación positiva entre la formación y la calidad profesional para cualquier ámbito, cómo no para el universitario. Con un mayor nivel de formación se entiende lógico pensar en mayores posibilidades para la actuación docente dentro del aula y, por extensión, para la mejora de la calidad de la enseñanza. Ahora bien, no debemos restringir la formación del profesorado al ámbito de los conocimientos sino ampliar esta visión con la incorporación de las competencias y habilidades como requisitos también necesarios. En el EEES el nuevo concepto de aprendizaje se opone a la mera transmisión de teoría por parte del docente y se amplía a la transmisión y establecimiento de competencias y habilidades en el alumnado a través del esfuerzo del docente. Por el motivo anterior, los cursos de formación en un sentido amplio ocupan un papel destacado dentro de las iniciativas para la mejora de la calidad docente universitaria.

Los proyectos de innovación docente también ocupan un lugar destacado dentro de las iniciativas para la mejora de la calidad de la docencia universitaria. En los últimos años se puede incluso afirmar que constituyen una herramienta fundamental para abordar la innovación productiva por las Universidades. Su objetivo es fomentar la innovación del profesorado, bien sea en grupos o de forma aislada, con el fin último de ofrecer una mejora o renovación de la práctica docente a través de la reflexión. Siguiendo a Pallisera et alt. (2010:11) resulta necesario que la cultura docente derivada del EEES incorpore la habitual reflexión sobre la práctica docente tanto individual como en grupo. Consideramos que precisamente los proyectos de innovación docente facilitan y permiten atender estas exigencias, de ahí su utilidad en este campo.

Finalmente, nos encontramos en un momento donde la enseñanza universitaria no puede ignorar la importancia de un segundo idioma. La incorporación al EEES justifica aún más la necesidad del idioma como una competencia transversal que posibilite 
tanto la movilidad de los estudiantes como el acceso a recursos bibliográficos de mayor alcance. La movilidad y el intercambio profesional también están pensados por el EEES para el profesor universitario. Le corresponde la labor no sólo de poseer la competencia de un segundo idioma, sino también la de motivar su adquisición en su alumnado. De ahí que las diferentes actividades de movilidad disponibles para el docente universitario ocupen también un lugar destacado dentro de las actuaciones de mejora de la calidad docente.

\section{METODOLOGÍA Y OBJETIVOS DE INVESTIGACIÓN}

El objetivo fundamental de este trabajo es proporcionar una visión general sobre las distintas iniciativas que las universidades andaluzas están realizando en materia de mejora de su calidad docente. Entendemos estas actuaciones en un sentido amplio que incorpora tanto la innovación docente como cualquier otra actividad encaminada a la mejora de la actuación académica universitaria. Este concepto así entendido es lo que generalmente se conoce bajo el término "formación docente".

Tras la incorporación al EEES y ante una repetida exigencia de calidad a las Universidades, consideramos necesario conocer qué medidas se están tomando para adecuar la actuación de los docentes a las nuevas demandas así como los principios que están guiando su puesta en práctica para cada caso concreto. De manera más específica nos planteamos conocer:

a) Objetivo 1: ¿̇qué principios guían las distintas iniciativas en materia de calidad docente? La existencia de una guía específica para el ámbito andaluz en estos temas nos lleva a interrogarnos en qué grado se está cumpliendo y siguiendo por las universidades andaluzas.

b) Objetivo 2: ¿̇qué iniciativas resultan más repetidas en materia de calidad docente dentro de las universidades andaluzas?. Nos planteamos ahora el análisis de la forma de aplicar la búsqueda de la calidad docente, qué actividades o funciones se realizan a tal fin, así como el órgano que se encarga de esta función dentro de la estructura universitaria.

c) Objetivo 3: ¿podría concretarse un Modelo para la Mejora de la Calidad Docente Andaluz? A tenor de los resultados obtenidos concluiremos si la homogeneidad encontrada nos permite hablar de un modelo andaluz en este tema.

Para dar respuesta a todos los interrogantes anteriores la metodología que hemos seguido en este trabajo ha abarcado dos fases. Una primera fase de revisión bibliográfica donde se ha analizado tanto la literatura existente sobre el tema como la información facilitada en cada una de las webs de las universidades andaluzas, prestando especial atención a la información referida a calidad docente. La segunda fase ha consistido en la elaboración de un cuestionario ' durante el mes de noviembre de 2008 que ha sido suministrado a los Secretariados o Vicerrectorados de Calidad u homólogos de todas las universidades andaluzas, un total de 9 Universidades -ver detalle en Tabla 2-. La tasa de respuesta obtenida ${ }^{2}$ tras el envío y después de insistir telefónicamente en cada una de las Universidades participantes ha sido del 55.5\%, 5 Universidades en términos absolutos.

\footnotetext{
1 El cuestionario será facilitado a toda aquella persona interesada.

2 Agradecemos el interés y la atención recibida por parte de las Universidades Andaluzas participantes en todo momento.
} 
TABLA 2. Población ObJeto de estudio

\begin{tabular}{|l|l|}
\hline Universidad & Web \\
\hline Universidad de Almería & http://www.ual.es/ \\
\hline Universidad de Cádiz & http://www.uca.es/ \\
\hline Universidad de Córdoba & http://www.uco.es/ \\
\hline Universidad de Granada & http://www.ugr.es/ \\
\hline Universidad de Huelva & http://www.uhu.es/ \\
\hline Universidad de Jaén & http://www.uja.es/ \\
\hline Universidad de Málaga & http://www.uma.es/ \\
\hline Universidad de Pablo de Olavide & http://www.upo.es/ \\
\hline Universidad de Sevilla & http://www.us.es/ \\
\hline
\end{tabular}

Fuente: Elaboración Propia

El cuestionario utilizado para esta investigación se compone de tres bloques donde se combinan diferentes escalas de medición que van desde respuestas múltiples y abiertas, utilizadas fundamentalmente para los bloques primero y tercero, a escalas Likert utilizadas, mayoritariamente, dentro del segundo bloque. Pasamos a comentar con mayor detalle el contenido del mismo.

Una cuestión que consideramos fundamental antes de profundizar en las actuaciones para la mejora de la calidad docente es la identificación del órgano encargado de estas funciones dentro del organigrama universitario, es decir, dar respuesta al interrogante ¿̇quién se encarga de la calidad docente? Por este motivo, un primer bloque de nuestro cuestionario estaba destinado a profundizar en la estructura y dimensión con la que cada Universidad participante se ocupa de las labores de formación e innovación docente, como funciones representativas del concepto calidad docente que manejamos en este trabajo. A continuación, el segundo bloque del cuestionario, de carácter más conceptual, ha quedado destinado a identificar los principios que rigen la calidad docente en cada Universidad participante. En tercer y último lugar, se ha incorporado un bloque tendente a determinar el contenido de las actividades que se están realizando en cada Universidad Andaluza para la mejora de la calidad docente según los siguientes apartados: formación docente, proyectos de innovación docente y apoyo a la movilidad.

Del análisis conjunto de los resultados obtenidos en todos los apartados del cuestionario se obtendrá la información necesaria para concluir sobre el tercer objetivo de nuestra investigación, la posibilidad de concretar un Modelo para la Mejora de la Calidad Docente Andaluz. 


\section{ANÁLISIS DE RESULTADOS3}

Abordamos en este último apartado el comentario de los principales resultados obtenidos para cada uno de los objetivos de investigación propuestos. Comenzaremos analizando el papel de la calidad docente dentro del organigrama universitario andaluz. A continuación se revisarán los principios de calidad utilizados por las universidades andaluzas así como el orden de preferencia de los mismos. Finalmente, concluiremos con el análisis de las actuaciones que realizan las universidades andaluzas para reforzar y mejorar la calidad de sus docentes.

\subsection{LA CALIDAD DOCENTE DENTRO DEL ORGANIGRAMA UNIVERSITARIO}

En un primer momento nos planteamos analizar si las funciones de formación e innovación docente, integradoras del concepto calidad docente que venimos estudiando, se agrupan bajo una misma unidad o dependen de unidades diferentes. A continuación revisaremos la dimensión operativa de tal unidad o unidades dentro de las Universidades participantes. Bajo nuestro punto de vista, ambos aspectos deben ser valorados para poder emitir un dictamen sobre la existencia o no de un Modelo Andaluz para la Mejora de la Calidad Docente, al permitirnos concluir sobre la existencia de semejanzas o divergencias en la visión y dimensión organizativa de la calidad docente dentro del entramado universitario.

Nuestros resultados evidencian que ambas fórmulas, gestión integrada o independiente, se utilizan por las universidades andaluzas, sobresaliendo la primera de ellas con un $60 \%$ de casos frente a un $40 \%$ restante que admite la gestión independiente de las labores de formación e innovación. Observamos cómo no existe una coherencia en este aspecto, cuestión que ya introduce un resultado negativo para el tercero de los objetivos que planteamos en esta investigación.

Igualmente solicitamos la denominación específica de la unidad o unidades encargadas de las labores de formación e innovación docente en cada Universidad para establecer semejanzas o divergencias en términos organizativos. Encontramos que la fórmula del Vicerrectorado específico era la menos elegida, $20 \%$ de los casos, y siendo los Secretariados o Unidades Técnicas, en idénticos porcentajes, las estructuras más repetidas en las Universidades participantes. Resulta llamativo observar cómo dentro de las denominaciones elegidas sólo en uno de los casos, concretamente se trataba de un Vicerrectorado, se menciona la calidad. En todos los casos restantes la denominación incluye referencias a la formación, innovación, docencia, entre otros, pero no se alude de manera explícita a este concepto.

Sobre la dimensión de las funciones de formación e innovación docente debemos comentar que, en términos generales, la dimensión física de la unidad, secretariado o vicerrectorado específico, entendiendo por ésta el número de empleados dedicados a estas actividades, se sitúa en término medio en 6 empleados. No podemos completar este dato con la dimensión económica, pues mayoritariamente no fue cumplimentado este ítem por las Universidades participantes.

\subsection{PRINCIPIOS ORIENTADORES DE LA CALIDAD DOCENTE}

El segundo bloque del cuestionario utilizado quedaba dedicado al análisis de los principios que dirigen en cada Universidad Andaluza las actuaciones en materia de

\footnotetext{
${ }^{3}$ El paquete estadístico utilizado para el tratamiento y análisis de datos ha sido SPSS V.17.0
} 
calidad docente. Ahora bien, ¿̇qué entendemos por principios de calidad?. Debido a que este estudio se adscribe al ámbito universitario andaluz y dado que para el mismo existe una guía de principios específica, CIDUA (Junta de Andalucía, 2005), éstos constituyen un ejemplo de principios de calidad con carácter muy específico al caso estudiado. No obstante, también se ha incluido dentro del cuestionario un ítem más amplio que hace referencia a los principios de calidad en general, es decir, al interés por la mejora de la calidad como elemento estratégico necesario en un entorno educativo cada vez más complejo y donde la diferenciación vía calidad puede resultar crucial para las diferentes Universidades.

Entendemos que las actuaciones en materia de calidad deben superar la visión individual para integrase en una estrategia conjunta. Peña (2003:8), refiriéndose a esta cuestión, indica que "los planes obedecerán a una propuesta de mejora de la institución que contemplará no sólo las necesidades de cada sujeto que enseña, sino también las preferencias y objetivos que corporativamente se buscan". Por este motivo se incluyó dentro del cuestionario un ítem relativo a las demandas del profesorado universitario en este tema, como colectivo especialmente interesado en la mejora de su calidad, otro ítem relativo al papel de las Políticas de Gobierno Universitario en general y de un modo específico al papel desempeñado, en su caso, por el Plan de Innovación y Formación Docente. Finalmente, se dejó abierta la pregunta mediante la incorporación de un ítem "otros", a detallar por cada Universidad participante si lo estimaba oportuno.

A la hora de evaluar este aspecto no sólo se incorporó en el cuestionario el listado de principios y elementos recogido por la Tabla 3 sino que también se solicitó un orden de prelación que nos indicase el interés hacia cada uno de estos principios. Es decir, del listado ofrecido se solicitaba una doble información; en primer lugar, determinar cuál o cuáles de estos elementos servían como base para orientar las actuaciones en materia de calidad docente, en segundo lugar, el orden de importancia concedido por las diferentes Universidades. En la Tabla 3 aparecen detallados los principales resultados obtenidos por cada uno de los ítems planteados.

TAbla 3. Principios orientadores de la Calidad docente. ANÁlisis de resultados (\%)

\begin{tabular}{|l|c|c|c|c|c|}
\hline $\begin{array}{l}\text { Valoración / } \\
\text { Principio } \\
\text { orientador }\end{array}$ & $\begin{array}{l}\text { Principios } \\
\text { de Calidad }\end{array}$ & $\begin{array}{l}\text { Principios de } \\
\text { Calidad } \\
\text { (CIDUA) }\end{array}$ & $\begin{array}{l}\text { Política de } \\
\text { Gobierno } \\
\text { Universitario }\end{array}$ & $\begin{array}{l}\text { Peticiones } \\
\text { del } \\
\text { Profesorado }\end{array}$ & $\begin{array}{l}\text { Plan de } \\
\text { Innovación y } \\
\text { Formación } \\
\text { Docente }\end{array}$ \\
\hline $\begin{array}{l}\text { Aparece } \\
\text { seleccionado } \\
\text { como relevante }\end{array}$ & 60 & 80 & 60 & 60 & 40 \\
\hline $\begin{array}{l}\text { Aparece } \\
\text { seleccionado } \\
\text { cómo el más } \\
\text { relevante }\end{array}$ & 20 & - & 20 & 20 & 40 \\
\hline $\begin{array}{l}\text { No aparece } \\
\text { seleccionado }\end{array}$ & 20 & 20 & 20 & 20 & - \\
\hline
\end{tabular}

Fuente: Elaboración Propia

Sólo uno de los elementos ofrecidos en el cuestionario, el relativo a los principios de calidad CIDUA (Junta de Andalucía, 2005), no resultó considerado por ninguna Universidad como el aspecto más importante, si bien, en el $80 \%$ de los casos se catalogó como importante para orientar las actuaciones en materia de calidad docente. Sí resultó seleccionado en un $20 \%$ de los casos como el principio más importante el ítem relativo a la utilización de principios de calidad en general. Idénticos resultados obtuvieron los ítems relativos a la Política de Gobierno Universitario 
y a las peticiones que el profesorado realiza. En el otro extremo, la existencia de un Plan de Innovación y Formación Docente fue el elemento más veces valorado como el más relevante, en un $60 \%$ de los casos, apareciendo en el $40 \%$ de los casos catalogado como importante, lo que significa que dentro de las Universidades participantes se apuntó su importancia o máxima importancia.

Si relacionamos el análisis de resultados efectuado con nuestro primer objetivo de investigación, la relación entre principios de calidad y actuaciones en esta materia para el ámbito universitario, podemos concluir sobre el papel fundamental del Plan de Innovación y Formación Docente. Aunque desde la teoría entendíamos que lo lógico sería que cada Universidad apostase por un Plan de Calidad amparándose a la hora de su redacción en principios de calidad y, específicamente, para el caso andaluz en las consideraciones ofrecidas por el informe CIDUA (Junta de Andalucía, 2005), nuestros resultados no han corroborado estas expectativas. La calidad y su importancia en un momento como el presente donde según Gvaramadze (2008) resulta ser el mayor reclamo de las Universidades en el EEES, no constituye el principio orientador por excelencia de las actuaciones en este tema. Más bien resulta ser la visión del equipo de gobierno de cada momento sobre este concepto, plasmadas en el Plan de Innovación y Formación de cada Universidad, el criterio más determinante.

\subsection{ACTIVIDADES DIRIGIDAS A LA MEJORA DE LA CALIDAD DOCENTE}

Como segundo objetivo de nuestra investigación nos propusimos analizar las actuaciones realizadas por las universidades andaluzas para la mejora de su calidad docente. Encontramos una amplia variedad de fórmulas para alcanzar este objetivo que van desde la tradicional financiación de cursos de formación hasta iniciativas más novedosas relacionadas con la innovación productiva, a la que anteriormente nos referíamos. Todas estas actuaciones que las Universidades están realizando quedan destinadas a mejorar los conocimientos, competencias y habilidades de sus docentes y tienen una nota común que descansa en el esfuerzo económico que supone su realización.

Bajo los fines de nuestro estudio y tras la revisión bibliográfica efectuada, las actividades destinadas a la mejora de la calidad docente dentro del ámbito universitario se pueden agrupar en torno a tres aspectos fundamentales.

- $\quad$ En primer lugar, la formación de los docentes. Resulta necesario que el docente disponga de una oferta de formación para su perfeccionamiento que le posibilite impulsar una mejora de su actuación. Nos referiremos a esta categoría como "Cursos de formación para el profesorado".

- En segundo lugar, la innovación. Exige un esfuerzo de las Universidades por impulsar las condiciones óptimas para que los docentes aborden su puesta en práctica. En este sentido, resulta habitual la existencia de algún tipo de financiación y apoyo a los proyectos que se embarcan en actividades innovativas, los "Proyectos de Innovación Docente".

- En tercer lugar, y debido a las exigencias de movilidad que se derivan de la implantación del EEES, se ha introducido un apartado relativo a las iniciativas destinadas a la "Movilidad del profesorado" que contemplan el análisis de la oferta de intercambio para el docente como vía de mejora de la calidad docente.

Pasamos a continuación el comentario de los resultados obtenidos en cada uno de estos apartados. 


\subsubsection{CURSOS DE FORMACIÓN DEL PROFESORADO}

El papel de la formación del profesorado no sólo se dirige a su perfeccionamiento sino también a su incorporación al puesto. El profesor novel o de nuevo ingreso y los cursos introductorios ocupan un lugar independiente en nuestro cuestionario por el motivo anterior. Específicamente, nos hemos preocupado por conocer si se realiza este tipo de formación inicial y si existen requisitos para optar a este tipo de formación. Nuestros resultados muestran que en todos los casos, un 100\%, se admite la existencia de cursos específicos para este colectivo dentro de las universidades andaluzas. En un $60 \%$ de los casos se demandan requisitos diferentes al propio hecho de contar con la condición de novel para su concesión, requisitos que las Universidades participantes no detallaron a pesar de dejar abierta esta pregunta dentro del cuestionario. Consideramos que el propio hecho de ser novel no necesitaría mayor justificación para optar a este tipo de cursos, si bien, debido a la falta de comentario al respecto no podemos extraer mejores conclusiones sobre este aspecto.

Centrándonos ahora en la formación no inicial de docentes, analizamos a continuación el contenido, número y duración de los cursos realizados para el perfeccionamiento de su profesorado. Con fines operativos y para poder sintetizar los resultados obtenidos, podemos distinguir cuatro grandes líneas de especialización docente en los momentos actuales. Nos referimos a las relativas al uso de nuevas tecnologías en la docencia; la centrada en la preparación para la enseñanza virtual; la destinada a la actualización metodológica $y$, finalmente, la que atiende a la formación en idiomas como competencia fundamental en el proceso de Bolonia. A todas ellas nos hemos referido en el cuestionario, encontrando como principales resultados los ofrecidos por el Gráfico n 1.

\section{GRÁFICO $1^{4}$. CONTENIDO DE CURSOS DE FORMACIÓN OFRECIDO POR LAS UNIVERSIDADES PARTICIPANTES}

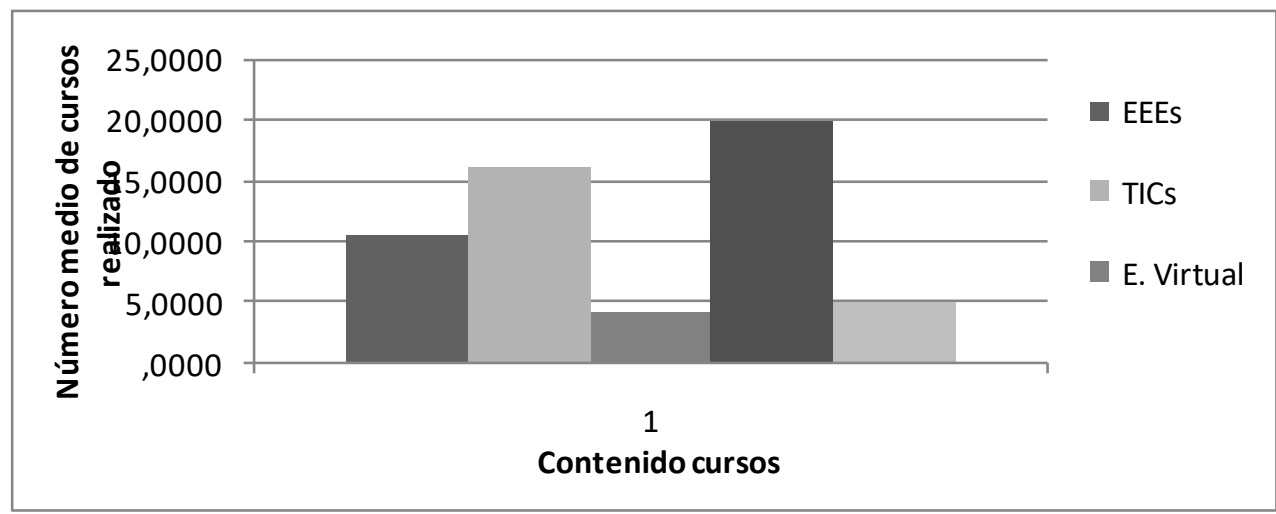

Fuente: Elaboración Propia

Como se puede observar, la distribución de las variables que recogen el número de cursos realizados según contenido no es homogénea, encontrándonos que los cursos relativos a la formación metodológica ocupan el primer puesto en el ranking de los ofertados por las universidades andaluzas. Para Villa (2008) "la puesta en práctica de la declaración de Bolonia requiere una modificación de la actuación docente en su concepción y metodologías para lograr los propósitos establecidos", cuestión que

\footnotetext{
4 Entiéndase: EEES: Espacio Europeo de Educación Superior, TICs: nuevas tecnologías de la información y comunicación, E. Virtual: aspectos relativos a docencia virtual, Metodología: nuevas herramientas metodológicas para la docencia e Idiomas: mejora del nivel de idioma.
} 
parece asumida por las Universidades participantes. Un segundo puesto ocupan los cursos relativos a la ayuda a docentes a implementar las nuevas tecnologías de la información y comunicación (TICS) dentro del aula, cuestión que se justifica ante el indudable papel de las mismas dentro de cualquier ámbito y, cómo no, en el de la enseñanza. Los blogs virtuales, webs, foros, chats, etc. constituyen ya parte del lenguaje universitario. El tercer puesto es para los contenidos de cursos relativos al EEES, todavía introductorios sobre la forma de organizar el nuevo escenario para educación superior y para ayudar a los docentes a entender y posibilitar su inmediata puesta en práctica.

Los contenidos menos ofertados por las Universidades participantes se relacionan tanto con la enseñanza virtual como con la formación relativa a idiomas que ocupa el último puesto dentro de nuestro ranking. La escasa implementación virtual de la docencia universitaria puede justificar el lugar ocupado por los cursos dedicados a este aspecto. Podemos justificar la distancia de esta posición con respecto a la obtenida por los cursos relativos a nuevas tecnologías TICs, con quienes comparten cierta similitud, por la aplicabilidad de los últimos, válidos no sólo para la docencia sino para la investigación y gestión universitaria. Finalmente, debemos comentar que consideramos alarmante que los cursos de idiomas hayan obtenido el último puesto en orden de relevancia, máximo si tenemos en cuenta la imperiosa necesidad de movilidad que impone el EEES.

Al igual que no ha resultado homogénea la distribución de cursos por contenido tampoco ha resultado con este carácter su duración que abarca desde las 3 horas hasta las 125 horas con independencia de su contenido, no pudiendo hablar de similitudes en este aspecto para ninguno de los casos tratados.

\subsubsection{PROYeCtOS DE INNOVACIÓN DOCENTE}

Los proyectos de innovación docente se encuentran presentes en todas las Universidades participantes, lo que nos permite concluir sobre su relevancia para el caso andaluz. Se trata de una iniciativa repetida para la mejora de la calidad docente en línea con los planteamientos de nuestro segundo objetivo de investigación. No obstante, se observan algunas diferencias mínimas en su puesta en práctica que pasamos a comentar.

No resulta la periodicidad de los proyectos de innovación docente una variable que difiera en el ámbito estudiado. En el $80 \%$ de los casos su carácter es anual, salvo en un solo caso que se establece un horizonte bianual para este tipo de proyectos.

Más diferencias encontramos en torno al objetivo al que van destinados los proyectos de innovación docente. En un $40 \%$ de los casos se dirigen exclusivamente a financiar actuaciones vinculadas específicamente a determinadas titulaciones mientras que en un $60 \%$ de los casos los destinatarios de estos proyectos son tanto titulaciones como asignaturas. Entendemos que lo anterior queda relacionado con la realización en grupo o individual de este tipo de proyectos. Iniciativas innovadoras para toda una titulación exigirán la participación de más de uno de sus profesores, mientras que para el caso de una asignatura sí puede ser un único docente suficiente para su implantación y desarrollo.

Sobre los criterios determinantes para la concesión de un proyecto de este tipo, sobresale en un $60 \%$ de los casos el grado de innovación de la propuesta presentada como criterio exclusivo para su selección por la Universidad. Este valor es fundamental ya que la propia naturaleza de los proyectos de innovación docente estriba en la capacidad de innovar y a través de la innovación, como ya se ha comentado, mejorar la calidad docente, con lo que no resulta de extrañar que sólo se exija 
"innovar". En el resto de los casos, la innovación y su presencia en la propuesta es un factor adicional al currículum académico de los participantes $-40 \%$, es decir, no aparece como un criterio exclusivo.

El presupuesto de los proyectos de innovación docente resulta inferior a 3.000 euros en un $60 \%$ de los casos estudiados. Para el $40 \%$ restante la financiación sí supera esta cantidad. Generalmente, no se trata de una cifra elevada, si bien, es cierto que la realización de estos proyectos se realiza dentro del marco de la infraestructura específica de cada Universidad con lo que se solapan algunos costes necesarios para su puesta en práctica reduciendo el presupuesto necesario.

A modo de resumen, consideramos especialmente relevante la presencia en todas las Universidades participantes de este tipo de proyectos al igual que estimamos como un factor de coherencia para el establecimiento de un Modelo Andaluz de Mejora de Calidad Docente el hecho de que las diferencias observadas no resulten significativas.

\subsubsection{MOVILIDAD DEL PROFESORADO}

La movilidad y el intercambio profesional también están pensados por el EEES para el profesor universitario. La Universidad debe establecer mecanismos que posibiliten la adquisición y el dominio de una segunda lengua, tanto por lo que respecta a las posibilidades de movilidad laboral como por lo que tiene que ver con las de expresión, comunicación y colaboración con las personas y con los pueblos (Junta de Andalucía, 2005:8). En esta línea de trabajo enfocamos el último bloque de nuestro cuestionario. Concretamente, nos ocupamos de conocer las características de las convocatorias específicas ${ }^{5}$ que las universidades andaluzas están efectuando para facilitar la movilidad de su profesorado.

Una primera cuestión que debemos comentar es que la presencia de este tipo de convocatorias se sitúa en el $80 \%$ de las Universidades participantes. Por tanto, no resulta una iniciativa tan extendida como el apoyo a la formación y a la innovación tratado con anterioridad. Quizás los resultados obtenidos pueden ser explicados por la tendencia creciente en los últimos años desde instituciones nacionales, europeas e internacionales a ofrecer ayudas con este carácter, circunstancia que puede actuar reduciendo la urgencia y necesidad de fomentar actuaciones propias desde cada Universidad.

No obstante, cuando este tipo de convocatorias se oferta por la Universidad suele poseer unas notas comunes. Generalmente, en un $66.7 \%$ de los casos, se trata de convocatorias con una periodicidad anual destinadas a financiar la movilidad de docentes no sólo a nivel europeo sino con carácter internacional -un $66.7 \%$ de los casos- y que consisten en una ayuda de tipo económico. Sobre los criterios establecidos para su concesión, sobresale el currículum de los solicitantes como elemento decisivo o determinante para su concesión -un $66.7 \%$ de los casos-.

La formación idiomática del docente resulta un aspecto vital en el entorno actual pues ya no resultan tan lejanos aspectos como la docencia en un segundo idioma, las iniciativas de intercambio docente, la utilización de recursos para la docencia en otro idioma, etc. Sin embargo, resultan llamativos los resultados obtenidos al respecto por cuanto asignan tanto el último lugar a las iniciativas de formación en idiomas como un panorama no excesivamente favorable en materia de convocatorias a la movilidad.

\footnotetext{
5 En este apartado no se incluyen las convocatorias oficiales establecidas tanto a nivel europeo, nacional como autonómico en este tema, sino que restringimos el análisis a la presencia de convocatorias exclusivas y adicionales a las anteriores por parte de las Universidades Andaluzas.
} 


\section{CONCLUSIONES}

Tras analizar las diferentes iniciativas realizadas por las universidades andaluzas en materia de mejora de su calidad docente, así como los principios que promueven su realización, podemos concluir nuestro trabajo afirmando que sí es posible hablar de un Modelo de Mejora para la Calidad Docente Andaluz pero con algunas limitaciones derivadas del "fondo", y no de la "forma", de las actuaciones que lo conformarían.

En esencia, para que se pueda confirmar este modelo se necesita constatar la relación existente entre las distintas iniciativas en materia de calidad docente y la presencia de unos principios comunes que guíen estas actuaciones, cuestión que constituye nuestro primer objetivo de investigación. En este sentido, los Principios de Calidad, de forma general, o el informe CIDUA (Junta de Andalucía, 2005), para el caso andaluz, serían desde la teoría las dos opciones con más posibilidades para fundamentar un Modelo de Mejora para la Calidad Docente Andaluz. Sin embargo, nuestros resultados ponen de manifiesto que son las propias universidades, a través de sus respectivas Políticas y Planes de Formación Docente, las que definen el marco conceptual de sus actuaciones en temas de calidad. La especificidad encontrada se opone a la generalidad que debiera estar presente en un modelo común.

Por otro lado, nuestros resultados evidencian que en las universidades andaluzas se repiten con carácter general tres iniciativas para la mejora de la calidad docente: las actuaciones relativas al establecimiento de cursos de formación, la financiación de proyectos de innovación docente y, con un menor grado de seguimiento, las ayudas a la movilidad. Así pues, podría afirmarse la existencia de unas actuaciones comunes en materia de calidad docente, aunque con pequeñas diferencias, cuestión que ha sido analizada conforme a nuestro segundo objetivo de investigación.

Con respecto al tercer objetivo de investigación, cabría hablar de un Modelo para la Mejora de la Calidad Docente Andaluz pero con ciertas limitaciones. La falta de coherencia con principios de calidad constituye el principal problema que debería considerar este modelo para su efectivo funcionamiento y posible estandarización en un futuro. La realización de actuaciones en materia de calidad docente sin guía alguna, simplemente por la demanda del profesorado o por la réplica de actuaciones llevadas a cabo por otras universidades cercanas, no permiten acercarnos en profundidad y con la seriedad que solicita este tema para el ámbito universitario. Resulta necesario reflexionar sobre el "fondo" antes de abordar la "forma".

Debido a que el estudio ha sido realizado en el ámbito universitario andaluz exclusivamente, siendo ésta nuestra principal limitación, consideramos como posibles líneas de investigación las siguientes. En primer lugar, la extensión al ámbito nacional del acercamiento realizado al tema de la calidad docente universitaria, lo que nos permitiría extraer conclusiones tanto sobre la existencia de un Modelo para la Mejora de la Calidad Docente Español, como sobre las semejanzas y/o divergencias con respecto al caso andaluz. En segundo lugar, también estimamos oportuno ampliar e incorporar la universidad privada al estudio, con el fin de establecer comparaciones sobre el papel de la calidad docente dentro del contexto público y privado.

\section{BIBLIOGRAFÍA}

ARRANZ VAL, P. [2007]: Los sistemas de garantía de calidad en la Educación Superior en España. Propuesta de un modelo de acreditación para las titulaciones de Grado en Empresa. Tesis Doctoral, Universidad de Burgos.

Bueno García, C. y EsCudero Escorza, T. [2005]: El profesorado universitario ante la innovación educativa. XII Congreso Nacional de Modelos de Innovación Educativa: 595-601. 


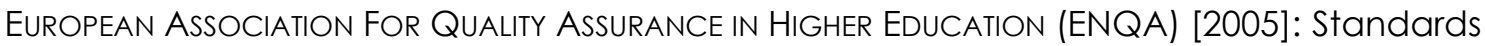
and Guidelines for Quality Assurance in the European Higher Education Area. Helsinki. Disponible en: http://www.enqa.eu/files/ENQA\%20Bergen\%20Report.pdf

GaLÁn, M. [2009]: Después de la calidad, la innovación. X Foro de Almagro "Los sistemas de información para la garantía de la calidad de las enseñanzas universitarias", octubre.

GVARAMADZE, I. [2008]: From quality assurance to quality enhancement in the European Higher Education Area. European Journal of Education, vol. 43, n 4: 443-455.

HARVEY, L., Y STENSAKER, B. [2008]: Quality culture: understandings, boundaries and linkages. European Journal of Education, vol. 43, n 4: 427-442.

JUNTA DE ANDALUCía (CIDUA) [2005]: Informe de la Comisión para la Innovación de la Docencia en las Universidades Andaluzas. Sevilla.

MARQUÉs, M. [2008]: Una experiencia madura de innovación educativa. Jornadas Nacionales sobre Estudios Universitarios de los Proyectos de Convergencia a la Realidad de los Nuevos Títulos, Universidad Jaume I, septiembre.

MAURI, T., COLL, C. Y OnRUBIA, J. [2004]: La evaluación de la calidad de los procesos de innovación docente universitaria. Una perspectiva constructivista. Revista de Docencia Universitaria $n^{\circ} 1: 1-11$.

MINISTERIO DE EDUCACIÓN Y CIENCIA [1992]: La formación del profesor universitario. M.E.C., Madrid.

Pallisera díaz, M., fullana Noell, J., Planas lladó, A. y del Valle Gómez, A. [2010]: la adaptación al espacio europeo de educación superior en España. Los cambios/retos que implica la enseñanza basada en competencias y orientaciones para responder a ellos. Revista Iberoamericana de Educación, $n^{\circ}$ 52/4: 1-13.

PEÑA, J.V. [2003]: Desarrollo profesional del docente universitario. Monografías virtuales $\mathrm{n}^{\circ} \quad 3 . \quad$ Disponible en: http://www.oei.es/valores2/monografias/monografia03/reflexion03.htm

PuLIDO, A. [2007]: La Universidad del siglo XXI. Introducción y reflexiones iniciales. IX A.N.E.C.A. Forum "La Universidad del siglo XXI": 17-26.

PuLIDO, A. [2009]: El futuro de la Universidad. Un tema para debate dentro y fuera de las Universidades. Delta Publicaciones, Madrid.

SÁNCHEZ NúÑEZ, J. [2001]: El desarrollo profesional del docente universitario. Revista Universidades, $\mathrm{n}^{\circ}$ 22: 1-11.

SEYMOUR, D. [1988]: Developing Academic Program: the climate for innovation. Higher regulation, self-study and program review in Higher Education. Columborg. Lemma.

VILLA, A. [2008]: La excelencia docente. Revista de Educación, n extraordinario 2008: $177-212$.

VIZCARRO, C. [2003]: Evaluación de la calidad de la docencia para su mejora Revista de la Red Estatal de Docencia Universitaria, vol. 3, n 1: 5-18. 